\title{
Tecnologias de Aprendizagem Colaborativa com Suporte Computacional Inclusivas ao Surdo: Um Mapeamento Sistemático de Literatura
}

\author{
Lucineide Rodrigues da Silva ${ }^{12}$, Laura Sánchez García ${ }^{1}$ \\ ${ }^{1}$ Programa de Pós-Graduação em Informática - Universidade Federal do Paraná (UFPR) \\ Curitiba - PR - Brasil \\ ${ }^{2}$ Câmpus do Pantanal - Universidade Federal do Mato Grosso do Sul (UFMS) \\ Corumbá - MS - Brasil \\ lucineide.silva@ufms.br, sg.laura@gmail.com
}

\begin{abstract}
Computational technologies can support collaborative learning and inclusion of Deaf students. To identify which technologies have been used in this context, a systematic literature mapping was executed. 28 studies were included among the 592 found. The results indicate that new researches must be developed, more attention should be given to the didactic approaches adopted, and video analysis should be exploited for the analysis of group interaction.
\end{abstract}

Resumo. Tecnologias computacionais podem apoiar a aprendizagem colaborativa e a inclusão de estudantes Surdos. Para identificar quais as tecnologias têm sido utilizadas neste contexto um mapeamento sistemático da literatura foi executado. Foram incluídos 28 estudos dentre os 592 encontrados. Os resultados indicam que novas pesquisas devem ser desenvolvidas, maior atenção deve ser dada às abordagens didáticas adotadas e a análise de vídeo deve ser explorada para a análise da interação dos grupos.

\section{Introdução}

A cultura Surda, segundo [Strobel 2013], abrange a língua, as ideias, as crenças, os costumes e os hábitos do povo Surdo. Por isso, tornar a escola inclusiva para promover uma educação bilíngue plena ao estudante Surdo demanda respeitar as diferenças culturais e a adoção de métodos de ensino e aprendizagem que atendam às suas necessidades, incluindo o uso de tecnologias educacionais adequadas.

A aprendizagem colaborativa, sob um ponto de vista amplo, foi definida por [Dillenbourg 1999] como "uma situação em que duas ou mais pessoas aprendem, ou tentam aprender algo juntas" (p. 8). No entanto, a aprendizagem colaborativa por si não é eficaz, uma vez que as interações sociais que levam à aprendizagem podem não ocorrer espontaneamente, sendo necessário que as tecnologias CSCL - Computer-Supported Collaborative Learning - as provoquem intencionalmente [Dillenbourg and Fischer 2007].

A CSCL é um campo multidisciplinar que compreende teoria para o entendimento da natureza dos processos de nível de grupo, necessários para uma aprendizagem colaborativa eficaz; metodologia, formas de analisar a construção de significado intersubjetivo que ocorre em interação dialógica em pequenos grupos; pedagogia, abordagens educacionais para estabelecer práticas de grupo que favoreçam a construção colaborativa do 
conhecimento; e tecnologia projetada para promover a cognição do grupo e apropriada para promover práticas de grupo desejáveis [Stahl et al. 2021].

Neste contexto, este mapeamento sistemático identifica quais são as tecnologias relatadas na literatura para apoiar o ensino e a aprendizagem colaborativa para/por estudantes Surdos. Este trabalho está organizado da seguinte forma: os trabalhos relacionados são apresentados na seção 2. A seção 3 traz o método adotado no mapeamento, e a seção 4, os resultados obtidos. Por fim, as discussões estão na seção 5 e as conclusões na 6 .

\section{Trabalhos Relacionados}

Não foram encontradas revisões ou mapeamentos sistemáticos que abordem exclusivamente a CSCL e estudantes Surdos. A revisão de [Aristizábal et al. 2017] identificou como a aprendizagem colaborativa foi utilizada na educação de crianças Surdas e que tipo de tecnologias eram utilizadas. A busca considerou publicações de 2012 a 2017, tendo selecionado 14 publicações para a extração de dados. Nesta revisão, as tecnologias adotadas para atingir o objetivo educacional era uma questão secundária.

Já a revisão de [Sousa et al. 2019] verificou a influência das tecnologias na educação de crianças Surdas ou com deficiência auditiva. Foram analisadas publicações entre 2010 e 2018, resultando na seleção de 21. Esta revisão abordou o mesmo público, porém, em apenas um dos estudos a tecnologia dava suporte à aprendizagem colaborativa.

Uma revisão sobre tecnologias de colaboração para crianças com deficiências foi conduzida por [Baykal et al. 2020]. No total, 35 publicações foram incluídas, e somente 2 se destinavam às crianças com deficiência auditiva. E, apenas a Biblioteca da ACM foi utilizada. A partir dos trabalhos relacionados foi possível identificar que um mapeamento sistemático que reúna e organize os estudos que tratem de tecnologias CSCL inclusivas ao Surdo pode preencher esse espaço e incentivar novas pesquisas sobre o tema.

\section{Estudo de mapeamento sistemático}

Este mapeamento foi revisado por ambas as autoras e seguiu as orientações de [Kitchenham and Charters 2007], com fases de planejamento, de execução, e divulgação.

\subsection{Planejamento}

O objetivo, baseado no paradigma GQM [Basili et al. 1994], é analisar estudos primários com o propósito de identificar e caracterizar as tecnologias de aprendizagem colaborativa com suporte computacional em relação à inclusão do Surdo do ponto de vista de pesquisadores da área de IHC, Informática na Educação, e engenheiros de software no contexto de publicações disponíveis nas bibliotecas digitais IEEE Xplore, ACM Guide to Computing Literature, Scopus e Web of Science.

Este mapeamento responde à questão de pesquisa: "Quais são as tecnologias de aprendizagem colaborativa com suporte computacional projetadas para incluir o Surdo?". Foram definidas subquestões para caracterizar cada tecnologia na Tabela 1. Para a definição dos termos, utilizou-se o PICO, indicado por [Kitchenham and Charters 2007], sem o item de comparação, uma vez que não estamos comparando alternativas. Selecionou-se os termos em Inglês para a string de busca conforme a Tabela 2.

Os critérios para a inclusão de uma publicação no conjunto de publicações para leitura são: CI1: Publicações que apresentem tecnologias CSCL inclusivas ao Surdo; CI2: 
Tabela 1. Subquestões para caracterizar cada tecnologia mapeada.

\begin{tabular}{|ll|}
\hline Q01 Qual o objetivo geral do estudo? & Q02 Qual a teoria CSCL utilizada no estudo? \\
\hline Q03 Qual o contexto da tecnologia? & Q04 Qual o objetivo educacional? \\
\hline Q05 Qual a abordagem pedagógica utilizada no estudo? & Q06 Qual o tipo de tecnologia? \\
\hline Q07 Como a tecnologia apoia a aprendizagem? & $\begin{array}{l}\text { Q08 Como ocorre a colaboração durante o uso da tecnolo- } \\
\text { gia? }\end{array}$ \\
\hline Q09 Como a tecnologia foi avaliada? & Q10 Quais aspectos da tecnologia foram avaliados? \\
\hline Q11 Quem avaliou? & Q12 Que técnica foi utilizada para a avaliação? \\
\hline $\begin{array}{l}\text { Q13 Qual o método de análise da interação em grupo utili- } \\
\text { zado no estudo? }\end{array}$ & Q14 Quais os recursos de acessibilidade disponibilizados? \\
\hline Q15 Quais recomendações / requisitos elencados para o projeto de tecnologias para apoio à aprendizagem colaborativa inclusivas para Surdos?
\end{tabular}

Tabela 2. Termos da string de busca

\begin{tabular}{|l|l|l|}
\hline Population & (deaf OR hearing impairment OR hearing impaired OR deafness) & AND \\
\hline Intervention & $\begin{array}{l}\text { ((collabora* OR coopera) AND (environment OR system OR software OR platform OR game OR } \\
\text { tool OR technology OR web OR online OR virtual OR internet OR mobile OR tangible) ) OR CSCL } \\
\text { OR groupware) }\end{array}$ & AND \\
\hline Outcome & (learn* OR teach* OR educat* OR knowledge) & \\
\hline
\end{tabular}

Publicações que apresentem metodologias para a concepção e/ou o desenvolvimento de tecnologias CSCL inclusivas ao Surdo.

Os critérios para exclusão de uma publicação do mapeamento são: CE1: Publicações cujo idioma seja diferente do Português ou Inglês; CE2: Publicações duplicadas; CE3: Publicações sem texto completo; CE4: Publicações não revisadas por pares (livros, índices e comentários); CE5: Publicações com estudos secundários ou terciários.

\subsection{Execução}

A execução das buscas para este mapeamento ocorreu em março de 2021. O processo de seleção das publicações ocorreu em duas etapas. A primeira, com a leitura dos títulos e resumos, para conforme os critérios de inclusão e exclusão, determinar se estavam no escopo do trabalho. Nos casos em que houve dúvidas quanto à exclusão na primeira etapa, a publicação seguiu para a próxima. A segunda etapa consistiu da leitura completa das publicações e da decisão final de inclusão ou exclusão conforme os critérios definidos.

A busca retornou 592 publicações, somadas as quatro bases. Na primeira etapa 51 publicações foram selecionadas. Destas, apenas 28 permaneceram após a leitura completa, como mostra a Tabela 3. As publicações, o critério de inclusão ou exclusão, o formulário e os dados extraídos estão em planilha do Google, disponível online ${ }^{1}$.

Tabela 3. Publicações retornadas na busca e selecionadas nos filtros.

\begin{tabular}{|lccccc|}
\hline & ACM & IEEE Xplore & Scopus & Web of Science & Total \\
\hline Retornados na Busca & 21 & 50 & 369 & 152 & 592 \\
\hline Selecionados no $1^{\circ}$ Filtro & 6 & 9 & 18 & 18 & 51 \\
\hline Selecionados no $2^{\circ}$ Filtro & 5 & 5 & 10 & 8 & 28 \\
\hline
\end{tabular}

\section{Resultados}

Ao todo foram incluídas 28 publicações neste mapeamento. Dentre os países dos quais pesquisas foram incluídas, o Brasil aparece com 6 publicações, seguido por Estados Unidos com 4, Portugal e Espanha com 3 publicações cada. China e Japão vem em seguida,

\footnotetext{
${ }^{1}$ https://bit.ly/3sC0KNa
} 
com 2 publicações cada. Dinamarca, Taiwan, Egito, Eslovênia, Kuwait, Colômbia, Jordânia e Namíbia com 1 publicação cada, ver Figura 1. A lista de países foi criada pela filiação dos autores. A seguir, apresentamos os achados pelas questões de pesquisa.
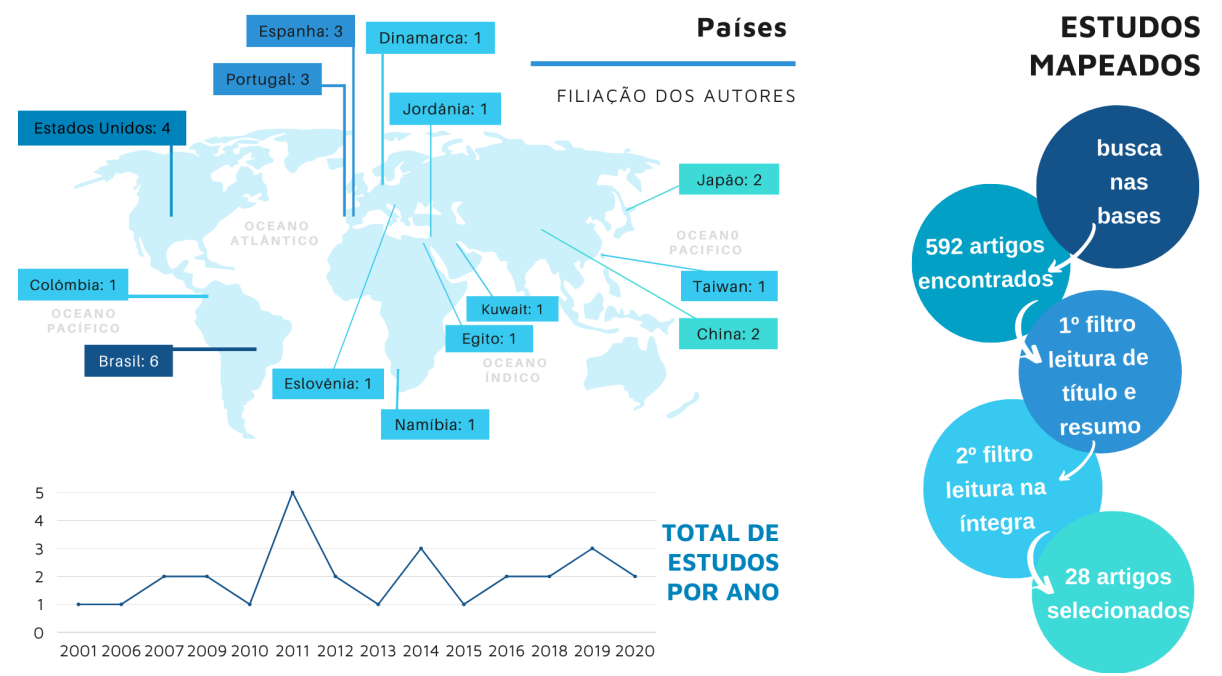

Figura 1. Visão geral dos estudos incluídos no mapeamento.

Q01 Qual o objetivo geral do estudo? O objetivo mais comum dos estudos encontrados foi o design de uma nova tecnologia. Para o design considerou-se o projeto e/ou o desenvolvimento. Foram 10 (35,7\%) estudos com design de tecnologias, de [Iversen et al. 2007], [Liu et al. 2007], [Nasr 2010], [Nobre et al. 2011], [Kosec et al. 2011], [Egusa et al. 2016], [Barata et al. 2019], [Paudyal et al. 2019], [Al-Jarrah 2019], e [Egusa et al. 2020]. Houve 8 (28,6\%) apropriações de alguma tecnologia já existente, em [Weiserbs 2001], [Beaton 2006], [Wu et al. 2009], [Apolonio et al. 2011], [Soeiro et al. 2011], [Soeiro et al. 2012], [Schley and Stinson 2016], e [Abiatal and Howard 2020].

São 7 (25\%) propostas de modelos/frameworks, caso de [Guimarães et al. 2011], [Trindade et al. 2013], [Cadeñanes and Arrieta 2014a], [Garnica and Arrieta 2014], [Cadeñanes and Arrieta 2014b], [Cano et al. 2018] e [Alsumait and Fasial 2018]. Registramos aqui que 3 publicações se referem ao modelo MuCy. Portanto, o mapeamento identificou o total de 5 diferentes modelos/frameworks. [Sheng and $\mathrm{Xu} 2009$ ] fez recomendações (3,6\%). [Trindade et al. 2012] identificou requisitos (3,6\%). [Oliveira et al. 2015] avaliou tecnologias (3,6\%).

Q02 Qual a teoria CSCL utilizada no estudo? Dentre os 28 estudos, 15 (53,6\%) não declararam em qual teoria se basearam, ou seja, a tecnologia foi desenvolvida sem uma teoria de suporte. A teoria do Construtivismo Social foi utilizada em 8 trabalhos (28,6\%), sendo eles [Iversen et al. 2007], [Wu et al. 2009], [Soeiro et al. 2011], [Soeiro et al. 2012], [Cadeñanes and Arrieta 2014a], [Garnica and Arrieta 2014], [Cadeñanes and Arrieta 2014b] e [Oliveira et al. 2015]. A teoria da criação do conhecimento foi utilizada por [Guimarães et al. 2011] e [Trindade et al. 2012] (7,1\%). O construtivismo em [Alsumait and Fasial 2018] e [Abiatal and Howard 2020] (7,1\%). A etnometodologia foi utilizada em [Trindade et al. 2013] (3,6\%).

Q03 Qual o contexto da tecnologia? Dos 28 estudos, 19 
(67,9\%) apoiam a aprendizagem em contexto escolar, sendo eles [Weiserbs 2001], [Beaton 2006], [Iversen et al. 2007], [Liu et al. 2007], [Sheng and Xu 2009], [Wu et al. 2009], [Apolonio et al. 2011], [Soeiro et al. 2011], [Soeiro et al. 2012], [Cadeñanes and Arrieta 2014a], [Garnica and Arrieta 2014], [Cadeñanes and Arrieta 2014b], [Egusa et al. 2016], [Schley and Stinson 2016], [Cano et al. 2018], [Paudyal et al. 2019], [Al-Jarrah 2019], [Egusa et al. 2020] e [Abiatal and Howard 2020]. Foram 6 (21,4\%) plataformas online, em [Nobre et al. 2011], [Guimarães et al. 2011], [Kosec et al. 2011], [Trindade et al. 2012], [Oliveira et al. 2015] e [Barata et al. 2019]. Não especificaram o contexto em 3 (10,7\%).

No contexto escolar foram encontradas tecnologias para apoio no ensino formal fundamental e superior. As plataformas online encontradas são para o ensino não formal. As de contexto não especificado são 2 modelos/frameworks e um ambiente virtual que podem ser adotados em diferentes contextos.

Q04 Qual o objetivo educacional? A aquisição de linguagem vem recebendo a maior atenção entre os estudos mapeados, correspondendo a 10 $(35,7 \%)$ estudos. [Cadeñanes and Arrieta 2014a], [Garnica and Arrieta 2014] e [Cadeñanes and Arrieta 2014b] apoiam a leitura, escrita fala e sinalização. [Cano et al. 2018], a leitura e escrita. [Iversen et al. 2007], a fala e linguagem, e [Egusa et al. 2016] a gramática. [Nobre et al. 2011] e [Oliveira et al. 2015] tratam da construção de termos em língua de sinais. [Guimarães et al. 2011] e [Trindade et al. 2012] apoiam a discussão e a aprendizagem da Libras.

Incluir o Surdo foi objetivo de 7 (25\%) estudos. [Weiserbs 2001] apoiou o compartilhamento do conhecimento adquirido na aula e a ampliação da percepção social um do outro. [Beaton 2006] e [Schley and Stinson 2016] apoiam a comunicação entre Surdos e ouvintes. [Wu et al. 2009] apoiam a colaboração em aulas de informática. [Apolonio et al. 2011] apoiam a interação por meio da escrita colaborativa. [Soeiro et al. 2011] e [Soeiro et al. 2012] apoiam a inclusão e a participação.

A aprendizagem de matemática é apoiada em [Liu et al. 2007] e [Abiatal and Howard 2020] (7,1\%). [Barata et al. 2019] apoia a criação de artes, [Egusa et al. 2020] a aprendizagem do crescimento e germinação de plantas, e [Kosec et al. 2011] a educação profissional, correspondendo a 3,6\% cada. Não foram especificados objetivos para $6(21,4 \%)$ das publicações, nas quais se enquadram ambientes virtuais de aprendizagem e modelos/frameworks que podem apoiar diversos objetivos.

Q05 Qual a abordagem pedagógica utilizada no estudo? Os estudos trazem uma descrição de como os estudantes devem usar a tecnologia e colaborar, porém em poucos casos uma referência ao método pedagógico é fornecida. $20(71,4 \%)$ estudos especificaram as práticas colaborativas do grupo, sendo o consenso a mais comum, aparecendo em [Guimarães et al. 2011], [Trindade et al. 2012]e [Egusa et al. 2016]. A escrita colaborativa ocorreu em [Apolonio et al. 2011] e [Schley and Stinson 2016]. A investigação em grupo em [Beaton 2006] e [Schley and Stinson 2016]. A aprendizagem baseada em projetos foi utilizada em [Soeiro et al. 2011] e [Soeiro et al. 2012]. A argumentação colaborativa em [Liu et al. 2007]. A resolução de problemas em pares em [Abiatal and Howard 2020]. Entrevista de três etapas e verificação por pares em [Al-Jarrah 2019], e o método invariante em [Cano et al. 2018]. 
Práticas onde cada estudante deveria compartilhar seu conhecimento e entendimento sobre determinado assunto, e na sequência, cada um deveria inserir sua resposta individual foram utilizadas em [Weiserbs 2001], [Iversen et al. 2007], [Nobre et al. 2011], [Cadeñanes and Arrieta 2014a], [Garnica and Arrieta 2014], [Cadeñanes and Arrieta 2014b], [Oliveira et al. 2015], [Egusa et al. 2020] (28,6\%). Outros $8(28,6 \%)$ dos estudos não especificaram a prática colaborativa adotada.

Q06 Qual o tipo de tecnologia? A tecnologia mais utilizada é o ambiente virtual de aprendizagem com 6 (21,4\%), em [Wu et al. 2009], [Nasr 2010], [Kosec et al. 2011], [Soeiro et al. 2011], [Soeiro et al. 2012] e [Al-Jarrah 2019]. Os jogos vem em seguida com $4(14,3 \%)$ em [Iversen et al. 2007], [Egusa et al. 2016], [Cano et al. 2018] e [Egusa et al. 2020]. Ambientes de realidade aumentada foram reportados em 3 (10,7\%) por [Cadeñanes and Arrieta 2014a], [Garnica and Arrieta 2014] e [Cadeñanes and Arrieta 2014b]. Sistemas de criação de conhecimento em [Guimarães et al. 2011] e [Trindade et al. 2012] (7,1\%).

Com 1 (3,6\%) dos estudos cada, o fórum de discussão de [Oliveira et al. 2015], realidade virtual de [Paudyal et al. 2019], ferramenta de visualização e anotação de [Liu et al. 2007], rede social de [Barata et al. 2019], e-mail de [Weiserbs 2001], software de exercícios de [Abiatal and Howard 2020], site de [Nobre et al. 2011], chat de [Beaton 2006], editor de texto de [Apolonio et al. 2011]. [Schley and Stinson 2016] utilizaram uma combinação de chat e editor colaborativo. Em 3 (10,7\%) dos estudos o tipo de tecnologia não é especificado por se tratarem de modelos e recomendações.

Q07 Como a tecnologia apoia a aprendizagem? [Jeong and Hmelo-Silver 2016] mapeou as 7 principais formas da tecnologia CSCL apoiar a aprendizagem colaborativa. Estas, foram utilizadas para categorizar esta resposta. Estabelecer uma tarefa colaborativa foi utilizada em [Iversen et al. 2007], [Apolonio et al. 2011], [Cadeñanes and Arrieta 2014a], [Garnica and Arrieta 2014], [Cadeñanes and Arrieta 2014b], [Egusa et al. 2016], [Cano et al. 2018], [Egusa et al. 2020] e [Abiatal and Howard 2020], totalizando $9(32,1 \%)$ estudos. Em 7 (25\%) houve uma junção de tarefa colaborativa, comunicação e compartilhamento de recursos, caso de [Wu et al. 2009], [Guimarães et al. 2011], [Kosec et al. 2011], [Soeiro et al. 2011], [Trindade et al. 2012], [Soeiro et al. 2012] e [Al-Jarrah 2019]. A tarefa colaborativa com a comunicação ocorreu em [Schley and Stinson 2016], [Barata et al. 2019] e [Paudyal et al. 2019] (10,7\%). O engajamento em co-construção em [Liu et al. 2007] e [Oliveira et al. 2015] (7,1\%). A comunicação foi utilizada em [Weiserbs 2001] e [Beaton 2006] (7,1\%). O compartilhamento de recursos sozinho ocorreu em [Nobre et al. 2011] (3,6\%), e em conjunto com a comunicação em [Nasr 2010] $(3,6 \%)$. Os $3(10,7 \%)$ não especificados correspondem a publicações que propõem modelos/frameworks e recomendações.

Q08 Como ocorre a colaboração durante o uso da tecnologia? A colaboração é principalmente face a face mediada, ocorrendo em 10 (35,7\%) dos estudos, [Beaton 2006], [Iversen et al. 2007], [Liu et al. 2007], [Cadeñanes and Arrieta 2014a], [Garnica and Arrieta 2014], [Cadeñanes and Arrieta 2014b], [Egusa et al. 2016], [Cano et al. 2018], [Egusa et al. 2020] e [Abiatal and Howard 2020]. A colaboração síncrona e assíncrona ocorreu em [Wu et al. 2009], [Nasr 2010], [Guimarães et al. 2011], [Kosec et al. 2011], [Apolonio et al. 2011] e [Trindade et al. 2012] (21,4\%). A colabo- 
ração assíncrona ocorre em [Weiserbs 2001], [Nobre et al. 2011], [Soeiro et al. 2011], [Soeiro et al. 2012] e [Oliveira et al. 2015] (17,9\%). A síncrona em [Paudyal et al. 2019] e [Al-Jarrah 2019] (7,1\%). [Schley and Stinson 2016] combinaram a colaboração síncrona com a face a face mediada por meio de um chat $(3,6 \%)$. Os $4(14,3 \%)$ não especificados são os 3 casos de modelos/frameworks e 1 trata-se de uma proposta de ambiente colaborativo que não especificou esta informação.

Q09 Como a tecnologia foi avaliada? Em 18 (64,3\%) estudos houve uma avaliação em contexto, sendo os de [Weiserbs 2001], [Beaton 2006], [Liu et al. 2007], [Iversen et al. 2007], [Wu et al. 2009], [Soeiro et al. 2011], [Apolonio et al. 2011], [Guimarães et al. 2011], [Soeiro et al. 2012], [Garnica and Arrieta 2014], [Cadeñanes and Arrieta 2014b], [Cadeñanes and Arrieta 2014a], [Oliveira et al. 2015], [Schley and Stinson 2016], [Egusa et al. 2016], [Cano et al. 2018], [Abiatal and Howard 2020] e [Egusa et al. 2020]. Para [Nobre et al. 2011] e [Kosec et al. 2011] $(7,1 \%)$ a avaliação foi em laboratório. Em $8(28,6 \%)$ dos estudos não houve referência a um procedimento de avaliação.

Q10 Quais aspectos da tecnologia foram avaliados? O aspecto considerado por [Weiserbs 2001], [Iversen et al. 2007], [Oliveira et al. 2015] e [Egusa et al. 2020] foi a experiência do usuário, totalizando $4(14,3 \%)$. A aprendizagem foi avaliada em [Liu et al. 2007], [Cadeñanes and Arrieta 2014a] e [Abiatal and Howard 2020] $(10,7 \%)$. Nos estudos de [Garnica and Arrieta 2014], [Cadeñanes and Arrieta 2014b] e [Egusa et al. 2016] (10,7\%) houve a avaliação de usabilidade e aprendizagem combinadas. Em [Soeiro et al. 2011] e [Soeiro et al. 2012] (7,1\%) a aprendizagem foi combinada com a colaboração. A colaboração em [Apolonio et al. 2011] e [Schley and Stinson 2016] (7,1\%). A usabilidade em [Nobre et al. 2011] e [Kosec et al. 2011] (7,1\%). [Beaton 2006] (3,6\%) avaliou a acessibilidade, [Guimarães et al. 2011] (3,6\%) a eficiência, e [Cano et al. 2018] (3,6\%) a performance. [Wu et al. 2009] (3,6\%) não deixou claro qual o aspecto foi avaliado.

Q11 Quem avaliou?
ram avaliação com especialistas. [Liu et al. 2007], [Wu et al. 2009], [Soeiro et al. 2011], [Guimarães et al. 2011], [Soeiro et al. 2012], [Garnica and Arrieta 2014], [Cadeñanes and Arrieta 2014b], [Cadeñanes and Arrieta 2014a], [Schley and Stinson 2016], [Cano et al. 2018] e [Abiatal and Howard 2020] tiveram apoio de especialistas. Em [Iversen et al. 2007], [Nobre et al. 2011], [Kosec et al. 2011], [Oliveira et al. 2015], [Egusa et al. 2016] e [Egusa et al. 2020], totalizando $6(21,4 \%)$ dos estudos contaram com a participação do usuário final. Em [Apolonio et al. 2011] (3,6\%) houve uma combinação de participação do usuário final com a avaliação do especialista. Em 11 estudos (39.3\%) os especialistas foram professores, sendo que em dois destes houve a combinação da participação dos pais com os professores como avaliadores.

Q12 Que técnica foi utilizada para a avaliação? O questionário foi a técnica mais comum, relatado em [Kosec et al. 2011], [Apolonio et al. 2011], [Cadeñanes and Arrieta 2014b], [Garnica and Arrieta 2014], [Cadeñanes and Arrieta 2014a] e [Egusa et al. 2020], com total de 6 (21,4\%) estudos. Em $3(10,7 \%)$ houve uma combinação de experimento e questionário, caso de [Nobre et al. 2011], [Oliveira et al. 2015] e [Egusa et al. 2016]. A observação também 
foi relatada em 3 (10,7\%), por [Beaton 2006], [Iversen et al. 2007] e [Liu et al. 2007]. [Soeiro et al. 2011] e [Soeiro et al. 2012] utilizaram a entrevista (7,1\%). O estudo de caso foi utilizado em [Cano et al. 2018] e [Guimarães et al. 2011] (7,1\%). A análise de documento em [Schley and Stinson 2016] (3,6\%). O uso de pré e pós testes em [Abiatal and Howard 2020] (3,6\%). Em [Weiserbs 2001] (3,6\%) foi relatado o uso de observação e análise de $e$-mail. [Wu et al. 2009] não especificou a técnica utilizada.

Q13 Qual o método de análise da interação em grupo utilizado no estudo? Em 21 (75\%) dos estudos não houve referência ao uso de análise da interação em grupo. A análise da conversação foi realizada em [Weiserbs 2001], [Soeiro et al. 2011], [Soeiro et al. 2012], [Trindade et al. 2013], [Schley and Stinson 2016] e [Abiatal and Howard 2020], totalizando 6 estudos $(21,4 \%)$. A análise da contribuição no texto colaborativo ocorreu em [Apolonio et al. 2011], 3,6\%.

Q14 Quais os recursos de acessibilidade disponibilizados? Em 20 (71,4\%) dos estudos não houve referência à disponibilização de recursos de acessibilidade. Em [Nasr 2010], [Kosec et al. 2011], [Guimarães et al. 2011] e [Oliveira et al. 2015] $(14,3 \%)$, a acessibilidade é alcançada através de vídeos com tradução de texto para sinais com o apoio de um intérprete, sendo que [Kosec et al. 2011] complementaram com um fórum de vídeo. Em [Paudyal et al. 2019] e [Trindade et al. 2012] $(7,1 \%)$ a tradução é feita em tempo real com o apoio de intérprete. Em [Al-Jarrah 2019] $(3,6 \%)$ a tradução é automática de voz para texto, de texto para sinal e de sinal para sinal. Em [Liu et al. 2007] $(3,6 \%)$ há quadrinhos ilustrando os problemas a serem resolvidos.

Q15 Quais recomendações / requisitos elencados para o projeto de tecnologias de apoio à aprendizagem colaborativa inclusivas para Surdos? Apenas 7 (25\%) publicações fizeram recomendações ou especificaram requisitos. As recomendações de [Wu et al. 2009] são disponibilizar módulos de troca de informações e discussão por chats e fóruns de discussão, registrar o histórico das discussões, fornecer testes e questionários com variados tipos de questões e em exibição aleatória para avaliação e feedback.

Em [Sheng and Xu 2009] é recomendado que em ambientes web colaborativos os professores deem feedback e forneçam soluções e orientações para os problemas que os estudantes encontrem em seu processo de aprendizagem. Aos designers e desenvolvedores, orientam em relação a que as necessidades desses estudantes sejam consideradas e para que se esforcem para atender à WCAG1.0. Por último, os estudantes devem ter o conhecimento e a habilidade necessários para o uso da tecnologia.

Conforme [Guimarães et al. 2011] é preciso fornecer colaboração com entrada de dados de forma síncrona e assíncrona em Língua de Sinais, discussão por ferramenta de vídeo multiusuário, protocolo de papéis e responsabilidades de cada participante, processo para tomada de decisão, armazenamento e compartilhamento das discussões e do conhecimento gerado, disponibilização de andaimes para novos membros, recuperação da informação em Sinais, e interface bilíngue (sinais e texto).

A partir dos desafios encontrados ao usar um sistema de criação de conhecimento, [Trindade et al. 2012] identificaram os requisitos: ter um vocabulário controlado, ter o envolvimento contínuo de um intérprete, ter um tesauro de termos técnicos, permitir apoio de intérprete remotamente, incluir tradução de fala para sinais e vice-versa, interface que permita interação baseada em Língua de Sinais, utilizar formas visuais, interação via re- 
conhecimento automático dos gestos em Língua de Sinais, uso de Signwriting para armazenamento e busca de informação, videoconferência interativa com múltiplos usuários, colaboração síncrona e assíncrona com tradução.

Já em [Trindade et al. 2013] os principais requisitos especificados são: disponibilizar ferramentas de suporte linguístico, prover uma forma de o intérprete mediar a comunicação, disponibilizar diferentes formas de apresentar informação, utilizar estratégias visuais para facilitar a percepção, usar papéis e responsabilidades para que a colaboração ocorra, oferecer formas de uso e recuperação da informação posterior às sessões síncronas, utilizar protocolos sociais e agentes conversacionais para guiar a comunicação.

Uma arquitetura com 5 pilares é proposta por [Alsumait and Fasial 2018]. No pilar Pedagógico, deve ser fornecido ao estudante o necessário para viabilizar a alfabetização. No pilar Espaço de Aprendizagem é preciso cuidar para que todos estejam envolvidos. Projetar adequadamente a interação social e a atividade, e promover comunicação multimodal. No pilar Tecnologia, incluir informações bilíngues (texto e sinal), materiais de vídeo, dicionário de Língua de Sinais, intérprete de Língua de Sinais e alternativas sincronizadas para multimídia. Para o pilar Comunicação, a ferramenta deve permitir a comunicação via Língua de Sinais e um protocolo de colaboração deve ser definido e descrito para o estudante. No pilar Avaliação deve-se considerar as áreas de alfabetização e linguagem, habilidade de escuta e concentração, habilidades sociais, e colaboração.

Um modelo para o desenvolvimento de jogos sérios para crianças com deficiência auditiva é proposto por [Cano et al. 2018]. O primeiro passo é estudar o perfil da criança, identificando os aspectos mais relevantes para dar suporte às atividades colaborativas. $\mathrm{O}$ segundo, definir quais aspectos devem ser considerados no desenho da interação, para que a colaboração ocorra nessa interação. O terceiro é a estruturação da colaboração, onde as atividades, os elementos e ferramentas de interação são definidas.

\section{Discussão}

Considerando que 28 estudos foram selecionados para este mapeamento, é possível notar que há espaço para novas pesquisas. Essa necessidade é evidenciada também pela abundante apropriação de tecnologias, chegando a $28,6 \%$ dos trabalhos. Como [Stahl et al. 2021] argumentam, as tecnologias que não foram projetadas para uso educacional possuem limitações e facilitam a distração.

As tecnologias têm sido utilizadas principalmente no ensino formal. A aquisição de linguagem é o uso mais frequente das tecnologias, refletindo uma das grandes dificuldades que o estudante surdo enfrenta em seu processo educacional, seguido pela inclusão, dificuldade encontrada quando precisam se comunicar com ouvintes em salas de aula inclusivas. Nos casos em que apoiam o ensino não formal, o objetivo mais comum é o aprofundamento nos estudos das língua de sinais, em geral para o público adulto.

Foram encontrados 5 modelos/frameworks para apoio ao projeto de tecnologias colaborativas. Apenas 2, propostos por [Trindade et al. 2013] e [Alsumait and Fasial 2018] apoiam projetos com objetivos mais genéricos. Estes utilizam teorias da CSCL que foram classificadas por [Stahl and Hakkarainen 2021] como intersubjetiva e subjetiva, respectivamente. [Stahl et al. 2021] consideram que as teorias com foco intersubjetivo são mais apropriadas para a pesquisa e a prática da CSCL. 
Destacamos aqui que para 53,6\% dos estudos não houve uma indicação de uso de teoria CSCL que suportasse a pesquisa. Inclusive, mesmo 71,4\% dos estudos descrevendo de alguma maneira as abordagens pedagógicas, foram raras as referências disponibilizadas sobre elas, indicando que deve ser dada maior atenção ao embasar a abordagem utilizada.

A variedade de tecnologias identificadas demonstra a riqueza de possibilidades para a inclusão do estudante Surdo em práticas colaborativas com a CSCL. Elas apresentam um uso predominante das interações face a face e poucos recursos de acessibilidade. Essa relação pode indicar que, por estarem todos no mesmo local, não haja essa necessidade. No entanto, o estudante Surdo se comunica via sinais e pode enfrentar dificuldades ao interagir com uma tecnologia que impõe o uso predominante de texto.

A experiência do usuário recebeu mais atenção durante a avaliação das tecnologias do que a própria aprendizagem. Ocorreu principalmente em contexto, com o apoio de especialistas, professores e, em poucos casos, pais. A análise da interação em grupo foi pouco utilizada, uma análise da conversação ocorreu em somente $21,4 \%$ dos estudos, todos analisando os registros escritos ou transcritos do que cada grupo produziu. Apenas o resultado da aprendizagem foi avaliado. Nenhum estudo fez análise de vídeos, do processo de aprendizagem e construção de conhecimento. Como ponderam [Schley and Stinson 2016], os vídeos permitiriam analisar a extensão e a qualidade da comunicação oral/gestual dos estudantes, e seria de grande valor para a pesquisa.

Os requisitos e recomendações encontrados foram agrupados. Há consenso dos autores quanto à necessidade de uso intenso da Língua de Sinais e os mais variados suportes linguísticos, tais como dicionários e intérprete. Também é preciso adotar um protocolo de colaboração, utilizar multimídia, representações visuais, andaimes, diferentes modalidades de comunicação, e o armazenamento e recuperação da informação gerada.

\section{Conclusões}

Este mapeamento sistemático da literatura baseou-se na análise de estudos primários com o objetivo de identificar e caracterizar tecnologias de aprendizagem colaborativa apoiada por computador inclusivas o Surdo. No total, 28 estudos foram incluídos. Os resultados apontam para a necessidade de novos estudos para o desenvolvimento de tecnologias. Apenas um framework, dentre os 5 encontrados, usa uma teoria CSCL classificada como intersubjetiva, apontada por [Stahl and Hakkarainen 2021] como a mais apropriada.

É preciso dar maior atenção e referenciar adequadamente as abordagens pedagógicas adotadas. A análise de vídeo, uma ferramenta potencialmente valiosa, não foi adotada. Os requisitos e recomendações para o desenvolvimento de tecnologias apontam para um consenso entre os pesquisadores que deve ser considerado.

Há limitações neste mapeamento. Não houve o uso de snowballing. As bases bibliográficas selecionadas podem não cobrir a totalidade de pesquisas publicadas. Por fim, há o risco de equívocos durante o processo de seleção e extração dos dados, mesmo com revisão por pares.

Como trabalhos futuros, utilizaremos destes resultados como insumo à oficinas participativas envolvendo estudantes Surdos para a construção de um ambiente colaborativo. Adicionalmente, esperamos que as oportunidades identificadas neste mapeamento motivem novos estudos primários e ampliem a inclusão do estudante Surdo. 


\section{Referências}

Abiatal, L. K. and Howard, G. R. (2020). Constructivism-led assistive technology: An experiment at a Namibian special primary school. South African Journal of Childhood Education, 10(1).

Al-Jarrah, A. A. (2019). Development of collaborative virtual learning environments for enhancing deaf people's learning in Jordan. In Advances in Intelligent Systems and Computing, volume 880, pages 1017-1028. Springer, Cham.

Alsumait, A. and Fasial, M. (2018). CLTD: Collaborative learning tool for Deaf. In ACM International Conference Proceeding Series, pages 146-151, New York, NY, USA. ACM.

Apolonio, S. O., De Deus, L. C., Borges, M. R., and Vivacqua, A. (2011). A collaborative approach for supporting interaction among hearing impaired and listeners. In CEUR Workshop Proceedings, volume 696, pages 52-56.

Aristizábal, L. F., Cano, S., Collazos, C. A., Solano, A., and Slegers, K. (2017). Collaborative learning as educational strategy for deaf children. In Proceedings of the XVIII International Conference on Human Computer Interaction, pages 1-8, New York, NY, USA. ACM.

Barata, A., Escudeiro, P., Duarte, V., and Lino, J. (2019). Inclusion through digital arts: Creating a community of practice. In Multi Conference on Computer Science and Information Systems, MCCSIS 2019 - Proceedings of the International Conferences on ICT, Society and Human Beings 2019, Connected Smart Cities 2019 and Web Based Communities and Social Media 2019, pages 145-152. IADIS Press.

Basili, V. R., Caldiera, G., and Rombach, H. D. (1994). The goal question metric approach. Encyclopedia of software engineering, pages 528-532.

Baykal, G. E., Van Mechelen, M., and Eriksson, E. (2020). Collaborative Technologies for Children with Special Needs. In Proceedings of the 2020 CHI Conference on Human Factors in Computing Systems, pages 1-13, New York, NY, USA. ACM.

Beaton, C. (2006). Work in progress: Tablet PC's as a leveling device! In Proceedings Frontiers in Education Conference, FIE, pages 15-16. IEEE.

Cadeñanes, J. and Arrieta, A. G. (2014a). Development of Sign Language Communication Skill on Children through augmented reality and the MuCy model. In Advances in Intelligent Systems and Computing, volume 292, pages 45-52. Springer, Cham.

Cadeñanes, J. and Arrieta, M. A. G. (2014b). Augmented reality: An observational study considering the MuCy model to develop communication skills on deaf children. In Polycarpou, M., de Carvalho, A. C. P. L. F., Pan, J.-S., Woźniak, M., Quintian, H., and Corchado, E., editors, International Conference on Hybrid Artificial Intelligence Systems HAIS 2014. Lecture Notes in Computer Science, number 7 in Lecture Notes in Computer Science, pages 233-240, Cham. Springer International Publishing.

Cano, S., Collazos, C. A., Aristizabal, L. F., Moreira, F., Peñeñory, V. M., and Agredo, V. (2018). Designing collaborative strategies supporting literacy skills in children with cochlear implants using serious games. In Advances in Intelligent Systems and Computing, volume 746, pages 1317-1326. Springer, Cham. 
Dillenbourg, P. (1999). What do you mean by "collaborative learning"? In Dillenbourg, P., editor, Collaborative learning: Cognitive and computational approaches, chapter 1 , pages 1-19. Elsevier, Oxford.

Dillenbourg, P. and Fischer, F. (2007). Basics of computer-supported collaborative learning. Zeitschrift für Berufs- und Wirtschaftspädagogik, 21:111-130.

Egusa, R., Komiya, N., Kusunoki, F., Mizoguchi, H., Namatame, M., and Inagaki, S. (2020). Development of a Learning-Support System for Science Using Collaboration and Body Movement for Hearing-Impaired Children: Learning Support for Plant Germination and Growth Conditions. In Miesenberger, K., Manduchi, R., Covarrubias Rodriguez, M., and Peáz, P., editors, 17th International Conference on Computers Helping People with Special Needs, pages 157-165, Cham. Springer International Publishing.

Egusa, R., Namatame, M., Sakai, T., Tamaki, H., Mizoguchi, H., Kusunoki, F., and Inagaki, S. (2016). Preparatory development of a collaborative/interactive learning game using bodily movements for deaf children. In Proceedings of IDC 2016 - The 15th International Conference on Interaction Design and Children, pages 649-653, New York, NY, USA. ACM.

Garnica, J. J. C. and Arrieta, M. A. G. (2014). Augmented reality Sign Language Teaching Model for deaf children. In Advances in Intelligent Systems and Computing, volume 290, pages 351-358. Springer, Cham.

Guimarães, C., Antunes, D. R., Fernandes, L. S. S., and Miranda, A. (2011). Empowering collaboration among the Deaf: Internet-based knowledge creation system. In Proceedings of the IADIS International Conference WWW/Internet 2011, ICWI 2011, pages 137-144.

Iversen, O. S., Kortbek, K. J., Nielsen, K. R., and Aagaard, L. (2007). Stepstone: An interactive floor application for hearing impaired children with a cochlear implant. In Proceedings of the 6th international Conference on Interaction Design And Children, IDC 2007, pages 117-124, New York, New York, USA. ACM Press.

Jeong, H. and Hmelo-Silver, C. E. (2016). Seven Affordances of Computer-Supported Collaborative Learning: How to Support Collaborative Learning? How Can Technologies Help? Educational Psychologist, 51(2):247-265.

Kitchenham, B. and Charters, S. (2007). Guidelines for performing systematic literature reviews in software engineering. Technical report, Software Engineering Group Department of Computer Science Keele University.

Kosec, P., Debevc, M., Kožuh, I., Rotovnik, M., and Holzinger, A. (2011). Accessible and collaborative moodle-based learning management environment for web users with varying degrees of hearing. In Proceedings of the 14th IASTED International Conference on Computers and Advanced Technology in Education, CATE 2011, pages 73-79, Calgary,AB,Canada. ACTAPRESS.

Liu, C. C., Tao, S. Y., Ho, K. W., Liu, B. J., and Hsu, C. C. (2007). Constructing an MCSCL groupware to improve the problem-solving experience of mathematics for hearing-impaired students. In Proceedings - The 7th IEEE International Conference on Advanced Learning Technologies, ICALT 2007, pages 345-347. IEEE. 
Nasr, M. M. (2010). An enhanced e-learning environment for Deaf/HOH pupils. In ICCTD 2010 - 2010 2nd International Conference on Computer Technology and Development, Proceedings, pages 724-727. IEEE.

Nobre, D. A., Ferreira, M., De Araújo, T. M. U., Nascimento, I. R., Carvalho, P., and Filho, G. L. (2011). WikiLIBRAS: Construção colaborativa de um dicionário multimídia em língua brasileira de sinais. In WebMedia 2011 - XVII Brazilian Symposium on Multimedia and the Web, co-located with XXV Brazilian Symposium on Database, SBBD 2011, pages 244-251, Porto Alegre, RS, Brasil. SBC.

Oliveira, F. C. M., Gomes, G. N., De Freitas, A. T., De Oliveira, A. C., Silva, L. C., and Queiroz, B. (2015). A comparative study of the acceptability of signs for the Brazilian sign language created in person and remotely. In SIGCSE 2015 - Proceedings of the 46th ACM Technical Symposium on Computer Science Education, pages 207211, New York, New York, USA. ACM Press.

Paudyal, P., Banerjee, A., Hu, Y., and Gupta, S. (2019). DAVEE: A deaf accessible virtual environment for education. In $C$ and $C 2019$ - Proceedings of the 2019 Creativity and Cognition, pages 522-526, New York, NY, USA. ACM.

Schley, S. and Stinson, M. (2016). Collaborative Writing in the Postsecondary Classroom: Online, In-Person, and Synchronous Group Work with Deaf, Hard-of-Hearing, and Hearing Students. The Journal of Postsecondary Education and Disability, 29(2):151164.

Sheng, L. H. and Xu, J. C. (2009). A research on web-based cooperative learning of hearing impaired university learner. In Proceedings of 2009 4th International Conference on Computer Science and Education, ICCSE 2009, pages 1576-1579. IEEE.

Soeiro, D., De Figueiredo, A. D., and Ferreira, J. A. G. (2011). The learning management system as a social mediator: A story with a happy ending. In Proceedings of the European Conference on Games-based Learning, volume 2, pages 788-793.

Soeiro, D., de Figueiredo, A. D., and Ferreira, J. A. G. (2012). Mediating diversity and affection in blended learning: A story with a happy ending. Electronic Journal of e-Learning, 10(3):339-348.

Sousa, C. S., Ferreira, D., and Rodrigues, C. L. (2019). Technologies For Educating Deaf Children- A Systematic Literature Review. In Anais do XXX Simpósio Brasileiro de Informática na Educação (SBIE 2019), page 1161. Brazilian Computer Society (Sociedade Brasileira de Computação - SBC).

Stahl, G. and Hakkarainen, K. (2021). Theories of CSCL. In Cress, U., Rosé, C., Wise, A., and Oshima, J., editors, International handbook of computer-supported collaborative learning. Springer International Publishing, New York, NY, USA.

Stahl, G., Koschmann, T., and Suthers, D. (2021). Computer-supported collaborative learning. In Sawyer, K. R., editor, Cambridge handbook of the learning sciences, chapter 21. Cambridge University Press, Cambridge, $3^{\text {a }}$ edition.

Strobel, K. (2013). As Imagens do Outro Sobre a Cultura Surda. Ed. da UFSC, Florianópolis, 3 edition. 
Trindade, D. d. F. G., Guimarães, C., Antunes, D. R., Sánchez Garcia, L., Lopes da Silva, R. A., and Fernandes, S. (2012). Challenges of knowledge management and creation in communities of practice organisations of Deaf and non-Deaf members: requirements for a Web platform. Behaviour \& Information Technology, 31(8):799-810.

Trindade, D. d. F. G., Guimaraes, C., and Garcia, L. S. (2013). Conceptual framework for design of collaborative environments: Cultivating communities of practices for deaf inclusion. ICEIS 2013 - Proceedings of the 15th International Conference on Enterprise Information Systems, 2:206-215.

Weiserbs, B. (2001). Social and academic integration using e-mail between children with and without hearing impairments. Computers in the Schools, 16(2):29-44.

Wu, L., Xu, J., and Qu, X. (2009). Exploration of Moodle-based collaborative learning for the Deaf. In IFCSTA 2009 Proceedings - 2009 International Forum on Computer Science-Technology and Applications, volume 2, pages 145-147. IEEE. 